\title{
The giant-impact origin of primitive mantle heterogeneities
}

\author{
MAO TANG ${ }^{1}$, YOU ZHOU ${ }^{1,3}$, YUN LIU $^{1,2,3}$, \\ ${ }^{1}$ Institute of Geochemisry, Chinese Academy of Sciences, \\ Guiyang 550081, China \\ ${ }^{2}$ International Center for Planetary Science, Chengdu \\ University of Technology, Chengdu 610059, China \\ ${ }^{3}$ CAS Center for Excellence in Comparative Planetology, \\ Hefei 230026, China \\ (E-mail: tangmao@vip.gyig.ac.cn, zhouyou@mail.gyig.ac.cn, \\ liuyun@vip.gyig.ac.cn)
}

There are several hypotheses about the formation of primitive or ancient mantle heterogeneities. Chemical differentiation is one of the major hypotheses. However, the chemical differentiation model has difficulties to explain a lot of unique isotope and element compositions observed for those primitive and ancient reservoirs, such as the negative ${ }^{182} \mathrm{~W}$ anomaly signal, the extremely low deltaD value with high $\mathrm{H}$ concentrations, the low $\mathrm{He}$ concentration but with high ${ }^{3} \mathrm{He}^{/ 4} \mathrm{He}$ ratio, etc. These reservoirs are confirmed very old ( $>4.5 \mathrm{Ga}$ ) by $\mathrm{Pb}$-isotope based chronology. They are not destroyed or disturbed later, which is based on the results of many other geochemical tracers. Here we propose that these reservoirs are even older than previous suggestions and they originate from the core.

Based on hundreds of smoothed particle hydrodynamics (SPH) simulations, we find that a special giant impact, which is called as the core-merging giant impact (CMGI), can bring a part of silicate mantle directly into the liquid iron core. This part of mantle will be tore up into pieces with the size from several to tens of kilometers due to the turbulence erosion of iron liquid. They can react with the iron liquid to certain extent under extremely high temperatures $(>5000 \mathrm{~K})$. Then due to density deficit, they will quickly go back to coremantle boundary (CMB) and stay discontinuously at the CMB. We suggest that those primitive mantle heterogeneities close to CMB are caused by these iron-contaminated silicates ejected out from the core. Our model can explain almost all the isotope and element data observed so far. The prediction of our model is that those primitive heterogeneities must be even older than the age of Moon. They can have many signals that only core materials will possess, including those haven't been reported yet. These primitive mantle heterogeneities provide a relatively direct way to study the chemistry of Earth's core, opening a window for future study on the chemistry of Earth's core with "real samples". 